\title{
ON THE EXTENSION OF MULTIMEASURES AND INTEGRATION WITH RESPECT TO A MULTIMEASURE
}

\author{
DIMITRIOS A. KANDILAKIS
}

(Communicated by Palle E. T. Jorgensen)

\begin{abstract}
We present a Caratheodory-Hahn-Kluvanek-type theorem for multimeasures whose values are weakly compact and convex subsets of a Banach space. Set functions obtained by integrating a bounded measurable function with respect to a multimeasure are also studied.
\end{abstract}

\section{Preliminaries}

Let $\Omega$ be a set, $\Sigma$ a field of subsets of $\Omega$, and $X$ a real Banach space. The following notations will be in use:

$$
\begin{aligned}
& P_{(b) f c}(X)=\{A \subseteq X: \text { nonempty, (bounded) closed, convex }\} \\
& P_{w k c}(X)=\{A \subseteq X: \text { nonempty, weakly compact, convex }\}
\end{aligned}
$$

A set function $M: \Sigma \rightarrow 2^{X} \backslash\{\varnothing\}$ will be strongly additive if for $\left\{A_{n}\right\}_{n \mathbb{N}} \subseteq \Sigma$ pairwise disjoint, the series $\sum_{n>1} x_{n}$ converges for any $x_{n} \in M\left(A_{n}\right)$. The range $M(\Sigma)$ of $M$ is the set $\bigcup_{A \in \Sigma} M(A)$. If $\Sigma$ is a $\sigma$-field, a multimeasure $M$ is a map $M: \Sigma \rightarrow P_{f c}(X)$ such that $M(\varnothing)=\{0\}$ and for $\left\{A_{n}\right\}_{n \in \mathbb{N}} \subseteq \Sigma$ pairwise disjoint we have

$$
M\left(\bigcup_{n \in \mathbb{N}} A_{n}\right)=\sum_{n \geq 1} M\left(A_{n}\right)
$$

There are several ways to interpret the sum of the sets in the right-hand side. In this work we will be using the following two:

(1) $M$ is a (strong) multimeasure if for any $x_{n} \in M\left(A_{n}\right)$ the series $\sum_{n \geq 1} x_{n}$ converges in the norm and

$$
\begin{array}{r}
\sum_{n \geq 1} M\left(A_{n}\right)=\left\{x \in X: x=\sum_{n \geq 1} x_{n}\right. \text { (unconditionally convergent), } \\
\left.x_{n} \in M\left(A_{n}\right), n \in \mathbb{N}\right\} .
\end{array}
$$

Received by the editors June 6, 1990 and, in revised form, February 9, 1991.

1980 Mathematics Subject Classification (1985 Revision). Primary 28B20.

Key words and phrases. Set valued function, measure, multimeasure, support function, selector, weak compactness, uniformly strongly additive. 
(2) $M$ is a weak multimeasure if for every $x^{*} \in X^{*}, A \rightarrow \sigma\left(x^{*}, M(A)\right)=$ $\sup _{x \in M(A)}\left(x^{*}, x\right)$ is a signed measure on $\operatorname{IR} \cup\{+\infty\}$.

The above two definitions are equivalent in the case of $P_{w k c}(X)$-valued multimeasures [3, Proposition 3].

Let $\Sigma$ be a $\sigma$-field and $M: \Sigma \rightarrow P_{f c}(X)$ a multimeasure. If $A \in \Sigma$, let $\pi$ be the collection of all finite $\Sigma$-partitions of $A$. We define:

$$
|M|(A)=\sup _{\pi} \sum_{A_{n} \in \pi} h\left(\{0\}, M\left(A_{n}\right)\right)
$$

where $h$ denotes the Hausdorff distance.

If $|M|(\Omega)<\infty$, then we say that $M(\cdot)$ is of bounded variation. Note that $|M|(\cdot)$ is itself and $\mathrm{IR}_{+}$-valued measure.

The space of all $X$-valued countably additive vector measures on $\Sigma$ will be denoted by $M(X)$. A measure $m \in M(X)$ is a selector of a multimeasure $M: \Sigma \rightarrow P_{f c}(X)$ if $m(A) \in M(A)$ for every $A \in \Sigma$. The set of selectors of $M$ will be denoted by $S_{M}$. Recall that $S_{M}$ is decomposable, i.e., for $A \in \Sigma$ and $\left(m_{1}, m_{2}\right) \in S_{M} x S_{M}$ the measure $X_{A} m_{1}+X_{c} m_{2} \in S_{M}$ (here $X_{A} m(B)=$ $m(A \cap B)$ for all $B \in \Sigma)$.

If $\mu: \Sigma \rightarrow \mathrm{IR}_{+}$is a countably additive measure and $M: \Sigma \rightarrow P_{f c}(X)$ a set function then $M$ will be called continuous with respect to $\mu(M<<\mu)$ if $\lim _{\mu(E) \rightarrow 0} h(\{0\}, M(E))=0$.

\section{EXTENDING A STRONGLY ADDITIVE SET FUNCTION ON A $\sigma$-FIELD}

In this section we provide the conditions for which a set function defined on a field $\Sigma_{0}$ can be extended to a multimeasure on the $\sigma$-field generated by $\Sigma_{0}$.

Before going into our main theorem we need some auxiliary results. For the following assume that $X$ is a real Banach space.

Proposition 2.1. Let $\Sigma_{0}$ be a field of subsets of a set $\Omega, M: \Sigma_{0} \rightarrow P_{f c}(X)$ a set function such that $M\left(\Sigma_{0}\right)$ is a relatively weakly compact subset of $X$, and the application $A \rightarrow \sigma\left(x^{*}, M(A)\right)$ is a finitely additive measure for every $x^{*} \in X^{*}$. Then $M$ is strongly additive.

Proof. Let $\left\{A_{n}\right\}_{n \geq 1} \subseteq \Sigma_{0}$ pairwise disjoint and $x_{n} \in M\left(A_{n}\right)$. Since $\sigma\left(x^{*}, M(\cdot)\right)$ is finitely additive we have $\sigma\left(x^{*}, M\left(\bigcup_{1}^{k} A_{n}\right)\right)=\sum_{1}^{k} \sigma\left(x^{*}, M\left(A_{n}\right)\right)$ for every $x^{*} \in X^{*}$, therefore $M\left(\bigcup_{1}^{k} A_{n}\right)=\sum_{1}^{k}\left(M\left(A_{n}\right)\right.$ which implies that $\sum_{1}^{k} x_{n} \in$ $M\left(\bigcup_{1}^{k} A_{n}\right) \subseteq M\left(\Sigma_{0}\right)$ which is weakly relatively compact, therefore each subseries $\sum_{k>1} x_{n_{k}}$ has a weakly convergent subseries. We will show that for every $x^{*} \in X^{*}$ the series $\sum_{n \geq 1}\left(x^{*}, x_{n}\right)$ converges. If not, then there exists $x_{0}^{*} \in X^{*}$ such that the series $\sum_{n \geq 1}\left(x_{0}^{*}, x_{n}\right)$ diverges. Therefore the series of the positive terms or the series of the negative terms of $\sum_{n \geq 1}\left(x_{0}^{*}, x_{n}\right)$ diverges, a contradiction, since each subseries of $\sum_{n>1} x_{n}$-and consequently each subseries of $\sum_{n \geq 1}\left(x_{0}^{*}, x_{n}\right)$-has a convergent subseries.

The conclusion now follows from Theorem IV.10.1 of [2].

Lemma 2.2. If $\Sigma_{0}$ is a field of subsets of $\Omega$ and $M: \Sigma_{0} \rightarrow 2^{X} \backslash\{\varnothing\}$ is a strongly additive set function then

(i) $\lim _{n \rightarrow+\infty} h\left(\{0\}, \quad \sum_{k=n}^{\infty} M\left(A_{k}\right)\right)=0$ for any $\left\{A_{n}\right\}_{n \geq 1} \subseteq \Sigma_{0}$ pairwise disjoint. If we also assume that the application $A \rightarrow \sigma\left(x^{*}, \bar{M}(A)\right)$ is a finitely additive measure for every $x^{*} \in X^{*}$ then 
(ii) $M\left(\Sigma_{0}\right)$ is a bounded subset of $X$.

If in addition $\sigma\left(x^{*}, M(\cdot)\right)$ is countably additive for every $x^{*} \in X^{*}$ then

(iii) the collection $\left\{\sigma\left(x^{*}, M(\cdot)\right): x^{*} \in X^{*},\left\|x^{*}\right\| \leq 1\right\}$ is a uniformly strongly additive family of measures.

Proof. (i) If $h\left(\{0\}, \sum M\left(A_{k}\right)\right)$ does not converge to 0 as $n \rightarrow \infty$ then there exist $\varepsilon>0$ and a subsequence $\left\{n_{i}\right\}_{i \in \mathbb{N}}$ of $\mathbb{N}$, such that $h\left(\{0\}, \sum_{k \geq n_{j}}^{\infty} M\left(A_{k}\right)\right)$ $\geq \varepsilon$. Therefore for each $i$ there exists $m_{i} \in \mathbb{N}$ and $x_{i} \in X$ with $\left\|x_{j}\right\| \geq \varepsilon / 2$ such that $x_{i} \in \sum_{k=n_{i}}^{m_{i}} M\left(A_{k}\right)$ (here $\sum_{n \geq 1} M_{n}=\left\{x=\sum_{n \geq 1} x_{n}: x_{n} \in M_{n}\right\}$ with $\left.M_{n} \subseteq X, n \in \mathbb{N}\right)$. But then the series $\sum_{i \geq 1} x_{n_{i}}$ diverges, a contradiction.

(ii) As in the proof of Proposition 2.1 we have that $M$ is finitely additive. If $M\left(\Sigma_{0}\right)$ is not bounded then there exists $\left\{A_{n}\right\}_{n \geq 1} \subseteq \Sigma$ such that $\left.h(\{0\}), M\left(A_{1}\right)\right)>1, A_{n} \subseteq A_{n+1} \quad \forall n \in \mathbb{N}$, and $h\left(\{0\}, M\left(A_{n+1}\right)\right)>$ $2 h\left(\{0\}, M\left(A_{n}\right)\right)$.

Take $x_{1} \in M\left(A_{1}\right)$ such that $\left\|x_{1}\right\| \geq 1$ and $y_{n} \in A_{n}$ such that $\left\|y_{n+1}\right\|>$ $2 h\left(\{0\}, M\left(A_{n}\right)\right)$ for $n \geq 1$. Then $y_{n+1}=y_{n+1}^{\prime}+x_{n+1}$ where $x_{n+1} \in B_{n+1}=$ $A_{n+1} \backslash A_{n}$ and $y_{n+1}^{\prime} \in A_{n}$. Therefore $\left\|x_{n+1}\right\| \geq h\left(\{0\}, M\left(A_{n}\right)\right) \geq 1$, which implies that the series $\sum_{n \geq 1} x_{n}$ diverges while $\left\{B_{n}\right\}_{n \geq 1} \subseteq \Sigma$ are pairwise disjoint, $x_{n} \in B_{n}$, and $M$ is strongly additive, a contradiction.

(iii)

$$
\begin{aligned}
\lim _{n \rightarrow+\infty} \sup _{\left\|x^{*}\right\| \leq 1}\left|\sum_{k=n}^{\infty} \sigma\left(x^{*}, M\left(A_{k}\right)\right)\right| & =\lim _{n \rightarrow+\infty} \sup _{\left\|x^{*}\right\| \leq 1}\left|\sigma\left(x^{*}, \sum_{k=n}^{\infty} M\left(A_{k}\right)\right)\right| \\
& =\lim _{n \rightarrow+\infty} h\left(\{0\}, \sum_{k=n}^{\infty} M\left(A_{k}\right)\right)=0
\end{aligned}
$$

which implies that the collection $\left\{\sigma\left(x^{*}, M(\cdot)\right): x^{*} \in X^{*},\left\|x^{*}\right\| \leq 1\right\}$ is a strongly countably additive family of countably additive measures on $\Sigma_{0}$.

Proposition 2.3. Let $\Sigma_{0}$ be a field of subsets of a $\Omega$ and $M: \Sigma_{0} \rightarrow P_{f_{c}}(X)$ a strongly additive set function such that the applications $A \rightarrow \sigma\left(x^{*}, M(A)\right)$ are countably additive real-valued measures for every $x^{*} \in X^{*}$. Then there exists a nonnegative real-valued countably additive measure $\mu$ on $\Sigma_{0}$ such that $M<<\mu$.

Proof. By Lemma 2.2(iii) the collection $\left\{\sigma\left(x^{*}, M(\cdot)\right): x^{*} \in X^{*}, \quad\left\|x^{*}\right\| \leq 1\right\}$ is a uniformly countably additive family of real-valued measures on $\Sigma_{0}$. By the Caratheodory extension theorem for each $x^{*} \in X^{*},\left\|x^{*}\right\| \leq 1$, there exists a unique countably additive measure $\sigma_{x^{*}}(\cdot)$ which extends $\sigma\left(x^{*}, M(\cdot)\right)$ on the $\sigma$-field $\Sigma$ generated by $\Sigma_{0}$. Lemma 1 on p. 26 of [1] guarantees that the family $\left\{\sigma_{x^{*}}(\cdot): x^{*} \in X^{*},\left\|x^{*}\right\| \leq 1\right\}$ is uniformly countably additive and Theorem 4 on p. 11 of [1] provides us with a nonnegative real-valued countably additive measure $\mu$ on $\Sigma$ such that $\lim _{\mu(E) \rightarrow 0} \sup _{\left\|x^{*}\right\| \leq 1} \sigma_{x} *(E)=0$ which implies that $M<<\left.\mu\right|_{\Sigma_{0}}$.

Proposition 2.4. Let $\Sigma$ be a $\sigma$-field of subsets of a set $\Omega$ and $M: \Sigma \rightarrow P_{w k c}(X)$ a multimeasure. Then $M(\Sigma)$ is a relatively weakly compact subset of $X$.

Proof. Let $x^{*} \in X^{*}$. The application $A \rightarrow \sigma\left(x^{*}, M(A)\right)$ is a signed countably additive measure on $\Sigma$. By the Hahn decomposition theorem there exist two disjoint sets $A, B$ in $\Sigma$ whose union is $\Omega$ such that $A$ is positive and $B$ is 
negative with respect to $\sigma\left(x^{*}, M(\cdot)\right)$. Clearly $\sigma\left(x^{*}, M(A)\right) \geq \sigma\left(x^{*}, M(C)\right)$ for every $C \in \Sigma$. Since $M(A)$ is weakly compact there exists $x_{0} \in M(A)$ such that $\left(x^{*}, x_{0}\right)=\sigma\left(x^{*}, M(A)\right)$. But then $\left(x^{*}, x_{0}\right)=\sigma\left(x^{*} M(\Sigma)\right)$ and the conclusion follows from the James theorem.

Proposition 2.5. Let $\Sigma_{0}$ be a field of subsets of $\Omega, \Sigma$ the $\sigma$-field generated by $\Sigma_{0}$, and $M_{0}: \Sigma_{0} \rightarrow P_{b f c}(X)$ a set function such that the applications $A \rightarrow$ $\sigma\left(x^{*}, M_{0}(A)\right), A \in \Sigma_{0}, x^{*} \in X^{*}$, are countably additive real-valued measures on $\Sigma_{0}$. If there exists a nonnegative real-valued countably additive measure $\mu$ on $\Sigma$ such that $M_{0}<<\left.\mu\right|_{\Sigma_{0}}$ then $M$ can be extended to a weak multimeasure $M: \Sigma \rightarrow P_{b f c}(X)$.

Proof. We supply $\Sigma$ with the pseudometric $d$ defined by $d\left(E_{1}, E_{2}\right)=$ $\mu\left(E_{1} \Delta E_{2}\right), E_{1}, E_{2} \in \Sigma$. The subspace $\Sigma_{0}$ is then dense in $\Sigma$ with respect to $d$. Since $M<<\left.\mu\right|_{\Sigma_{0}}$ the measures $\sigma\left(x^{*}, M_{0}(\cdot)\right): \Sigma_{0} \rightarrow$ IR are uniformly continuous on $\Sigma_{0}$ for every $x^{*} \in X^{*}$. So each of them has a uniformly continuous extension $\sigma_{x^{*}}(\cdot): \Sigma \rightarrow$ IR. It is easily verified that $\sigma_{x^{*}}(\cdot)$ is a countably additive $\mu$-continuous measure on $\Sigma$ for every $x^{*} \in X^{*}$. Since the application $x^{*} \rightarrow \sigma\left(x^{*}, M(A)\right)$ is weakly lower semicontinuous for every $A \in \Sigma_{0}, \quad \Sigma_{0}$ is dense in $\Sigma$, and $\sigma_{x} *(\cdot)$ are uniformly continuous for every $x^{*} \in X^{*}$ we easily deduce that $x^{*} \rightarrow \sigma_{x} *(A)$ is also weakly lower semicontinuous for every $A \in \Sigma$. We now define $M: \Sigma \rightarrow P_{b f c}(X)$ by $M(A)=\left\{x \in X:\left(x^{*}, x\right) \leq \sigma_{x} *(A)\right.$ for every $\left.x^{*} \in X^{*}\right\}, A \in \Sigma$. The remarks on p. 4.8 of [5] guarantee that $M$ is a weak multimeasure. Obviously $M$ extends $M_{0}$ to $\Sigma$.

We are now ready to state the basic result of this section.

Theorem 2.6. Let $\Sigma_{0}$ be a field of subsets of a set $\Omega$ and $\Sigma$ the $\sigma$-field generated by $\Sigma_{0}$. If $M_{0}: \Sigma_{0} \rightarrow P_{w k c}(X)$ is a set function such that the set $\left\{\sigma\left(x^{*}, M_{0}(\cdot)\right)\right.$ : $\left.x^{*} \in X^{*},\left\|x^{*}\right\| \leq 1\right\}$ is a family of countably additive measures on $\Sigma_{0}$, then the following are equivalent:

(i) $M_{0}$ can be extended to a multimeasure $M: \Sigma \rightarrow P_{w k c}(X)$.

(ii) There exists a nonnegative real-valued countably additive measure $\mu$ on $\Sigma_{0}$ such that $M_{0}<<\mu$.

(iii) $M_{0}$ is strongly additive.

(iv) $M_{0}\left(\Sigma_{0}\right)$ is a telatively weakly compact subset of $X$.

Proof. We only have to show that (i) implies (iv)

Clearly $M_{0}\left(\Sigma_{0}\right)=M\left(\Sigma_{0}\right) \subseteq M(\Sigma)$. By Proposition $2.4 M(\Sigma)$ is relatively weakly compact so $M_{0}(\Sigma)$ is also relatively weakly compact.

\section{The INTEgRAL OF A BOUNDED MEASURABLE FUNCTION WITH RESPECT TO A MULTIMEASURE}

In this section we define the integral of a bounded measurable function with respect to a multimeasure and study its properties.

Assume that $(\Omega, \Sigma)$ is a measurable space, $X$ a Banach space, and $G: \Sigma \rightarrow$ $P_{w k c}(X)$ a multimeasure. Let $B(\Sigma)$ be the space of all functions $f: \Omega \rightarrow$ IR which are $\Sigma$-measurable and bounded.

Definition 3.1. The integral of $f \in B(\Sigma)$ with respect to $G$ on $A \in \Sigma$ is

$$
\int_{A} f d G=\left\{\int_{A} f d m: m \in S_{G}\right\} .
$$


Remark. By Theorem 1 of [3] we know that $S_{G}$ is not empty and Lemma 3 on p. 95 of [4] guarantees that the integrals on the right-hand side are well defined.

Theorem 3.2. If $G: \Sigma \rightarrow P_{w k c}(X)$ is a multimeasure of bounded variation and $f \in B(\Sigma)$ then the set function defined by $M(A)=\int_{A} f d G$ for $A \in \Sigma$ is a $P_{w k c}(X)$-valued multimeasure of bounded variation.

Proof. First we will show that $M(A) \in P_{w k c}(X)$ for every $A \in \Sigma$. So let $\left\{\int_{A} f d m_{n}\right\}_{n \in \mathbb{N}}$ be a sequence in $M(A)$ where $\left\{m_{n}\right\}_{n \in \mathbb{N}} \subseteq S_{G}$. Since $S_{G}$ is compact for the topology of simple weak pointwise convergence (see $[6, \S 4]$ ) there exists a subsequence $\left\{m_{n_{k}}\right\}_{k \in \mathbb{N}}$ of $\left\{m_{n}\right\}_{n \in \mathbb{N}}$ which converges to $m \in S_{G}$. But then for $x^{*} \in X^{*}$ we have:

$$
\begin{aligned}
\lim _{k \rightarrow+\infty}\left(x^{*}, \int_{A} f d m_{n_{k}}\right) & =\lim _{k \rightarrow+\infty} \int_{A} f d\left(x^{*}, m_{n_{k}}\right) \\
& =\int_{A} f d\left(x^{*}, m\right)=\left(x^{*}, \int_{A} f d m\right)
\end{aligned}
$$

(see [2, Example IV.13.18, p. 340]) which implies that $\int_{A} f d m_{n_{k}} \rightarrow \int_{A} f d m$. Therefore $M(A)$ is a weakly compact subset of $X$.

Now let $\left\{A_{n}\right\}_{n \in \mathbb{N}} \subseteq \Sigma$ pairwise disjoint. Then for $x^{*} \in X^{*}$ we have

$$
\begin{aligned}
\sigma\left(x^{*}, M\left(\bigcup_{1}^{k} A_{n}\right)\right) & =\sup _{m \in S_{G}}\left(x^{*}, \sum_{n=1}^{k} \int_{A_{n}} f d m\right) \\
& \leq \sum_{1}^{k} \sup _{m \in S_{G}}\left(x^{*}, \int_{A_{n}} f d m\right) \\
& =\sum_{1}^{k} \sigma\left(x^{*}, M\left(A_{n}\right)\right) .
\end{aligned}
$$

For $n=1,2, \ldots, k$ there exists $m_{n} \in S_{G}$ such that $\sigma\left(x^{*}, M\left(A_{n}\right)\right)$ $=\left(x^{*}, \int_{A_{n}} f d m_{n}\right), m_{n} \in S_{G}$, because $M\left(A_{n}\right)$ is $w$-compact. Since $S_{G}$ is decomposable the measure $m: \Omega \rightarrow X$ defined by $m=X_{A_{1}} m_{1}+\cdots+X_{A_{k-1}} m_{k-1}+$ $X_{\Omega-\bigcup_{i=1}^{k-1} A_{i}} m_{k}$ is a selector of $G$, therefore

$$
\left(x^{*}, \int_{\bigcup_{1}^{k} A_{j}} f d m\right)=\sum_{1}^{k}\left(x^{*}, \int_{A_{j}} f d m_{j}\right)=\sum_{1} \sigma\left(x^{*}, M\left(A_{j}\right)\right)
$$

so

$$
\sigma\left(x^{*}, M\left(\bigcup_{1}^{k} A_{i}\right)\right) \geq \sum_{i=1}^{k} \sigma\left(x^{*}, M\left(A_{i}\right)\right) .
$$

By (1) and (2) above we get that

$$
\sigma\left(x^{*}, M\left(\bigcup_{1}^{k} A_{i}\right)\right) \geq \sum_{i=1}^{k} \sigma\left(x^{*}, M\left(A_{i}\right)\right) .
$$


Since $\bigcup_{i=1}^{\infty} A_{i}=\left(\bigcup_{i=1}^{k} A_{i}\right) \bigcup\left(\bigcup_{i=k+1}^{\infty} A_{i}\right)$ we have

$$
\begin{gathered}
\left|\sigma\left(x^{*}, M\left(\bigcup_{1}^{\infty} A_{i}\right)\right)-\sigma\left(x^{*}, M\left(\bigcup_{1}^{k} A_{i}\right)\right)\right|=\left|\sigma\left(x^{*}, M\left(\bigcup_{k+1}^{\infty} A_{i}\right)\right)\right| \\
=\left|\sup _{m \in S_{G}}\left(x^{*}, \int_{\bigcup_{k+1}^{\infty} A_{i}} f d m\right)\right| \leq\|f\|_{\infty} \cdot v\left(\sigma\left(x^{*}, G\left(^{\prime}\right)\right) \bigcup_{k+1}^{\infty} A_{i}\right)
\end{gathered}
$$

which converges to 0 as $k \rightarrow+\infty$ since $G$ is of bounded variation (here $v\left(\sigma\left(x^{*}, G(\cdot)\right), A\right)$ denotes the variation of $\sigma\left(x^{*}, G(\cdot)\right)$ on $\left.A \in \Sigma\right)$. Therefore $M$ is a multimeasure. We can show that $M$ is of bounded variation by an easy computation.

We will now compute the support function of $M$. Let $f^{+}=\max (f, 0)$ and $f^{-}=\max (-f, 0)$.

Theorem 3.3. If $G$ is as above then for $A \in \Sigma$

$$
\sigma\left(x^{*}, M(A)\right)=\int_{A} f^{+} d \sigma\left(x^{*}, G(\cdot)\right)-\int_{A} f^{-} d \sigma\left(-x^{*}, G(\cdot)\right) .
$$

Proof. First assume that $f$ is a simple function: $f=\sum_{1}^{k} a_{i} X_{A_{i}}+\sum_{1}^{\lambda} b_{j} X_{B_{j}}$ where $a_{i}>0, i=1,2, \ldots, k, b_{j}<0, j=1,2, \ldots, \lambda$, and $A_{i} \cap B_{j}=\varnothing$ for $i=1, \ldots, k, j=1, \ldots, \lambda$. Then for $C=\bigcup_{1}^{k} A_{i}$ and $D=\bigcup_{1}^{\lambda} B_{j}$ we have

$$
\begin{aligned}
\sigma\left(x^{*}, M(A)\right)= & \left.\sigma\left(x^{*}, \int_{A} f d G\right)\right) \\
= & \sigma\left(x^{*}, \int_{C} f d G\right)+\sigma\left(x^{*}, \int_{D} f d G\right) \\
= & \sup _{m \in S_{G}}\left(x^{*}, \int_{C} f d m\right)+\sup _{m \in S_{G}}\left(x^{*}, \int_{D} f d m\right) \\
= & \sup _{m \in S_{G}}\left(x^{*}, \sum_{1}^{\lambda} a_{i} m\left(A_{i} \cup A\right)\right) \\
& +\sup _{m \in S_{G}}\left(-x^{*}, \sum_{1}^{\lambda}\left(-b_{j}\right) m\left(B_{j} \cap A\right)\right) \\
= & \int_{A} f^{+} d \sigma\left(x^{*}, G(\cdot)\right)-\int_{A} f^{-} d \sigma\left(-x^{*}, G(\cdot)\right)
\end{aligned}
$$

and the result follows from a density argument.

We will now study the relation between $S_{M}$ and $S_{G}$. So let $m \in S_{G}$. Then measure $n: \Sigma \rightarrow X$ defined by $n(A)=\int_{A} f d m$ for $A \in \Sigma$ is clearly a selector of $M$. Conversely let $n \in S_{M}$ and for $A \in \Sigma$ define

$$
F(A)=\left\{m(A): m \in S_{M} \text { and } n(A)=\int_{A} f d m\right\} .
$$

Clearly $F(A)$ is not empty for every $A \in \Sigma$ and it is easy to show that $F: \Sigma \rightarrow P_{w k c}(X)$ is also a multimeasure. By Theorem 1 of [3] $F$ has a selector $m$ which clearly satisfies $n(A)=\int_{A} f d m$ for every $A \in \Sigma$.

So we have proved the following. 
Theorem 3.4. Let $M, G$, and $f$ be as above. Then:

(i) If $m \in S_{G}$ the measure $n$ defined by $n(A)=\int_{A} f d m$ is a selector of $M$.

(ii) If $n \in S_{M}$ then there exists $m \in S_{G}$ such that $n(A)=\int_{A} f d m$ for every $A \in \Sigma$.

In order to study the extreme structure of $S_{M}$ we need the following lemma.

Lemma 3.5. If $f \in B(\Sigma), f(w) \neq 0$ for every $w \in \Omega, m \in M(X)$, and $\int_{A} f d m=0$ for every $A \in \Sigma$, then $m$ is identically zero on $\Sigma$.

Proof. Suppose that there exists $A \in \Sigma$ such that $m(A) \neq 0$. Then either $m\{w \in A: f(w)>0\} \neq 0$ or $m\{w \in A: f(w)<0\} \neq 0$. Without loss of generality assume $m\{w \in A: f(w)>0\} \neq 0$. Let $B=\{w \in \Omega: f(w)>0\}$.

For $n \in \mathbb{N}$ define $A_{n}=\left\{w \in B: f(w)>\frac{1}{n}\right\}$. Then $\bigcup_{1}^{\infty} A_{n}=B$. Since $m(B)>0$ there exists $k \in \mathbb{N}$ such that $m\left(A_{k}\right) \neq 0$. Take $x^{*} \in X^{*}$ such that $\left(x^{*}, m\left(A_{k}\right)\right) \neq 0$. Let $\left(x^{*}, m(\cdot)\right)^{+}$and $(x, m(\cdot))^{-}$be the Jordan decomposition of $\left(x^{*}, m(\cdot)\right)$ and $B_{1}, B_{2}$ the corresponding Hahn decomposition of $A_{k}$. Then either $\left(x^{*}, m\left(B_{1}\right)\right)^{+} \neq 0$ or $\left(x^{*}, m\left(B_{2}\right)\right)^{-} \neq 0$. Without loss of generality assume that $\left(x^{*}, m\left(B_{1}\right)\right)^{+} \neq 0$. Then

$$
0=\left(x^{*}, \int_{B_{1}} f d m\right)=\int_{B_{1}} f d\left(x^{*}, m(\cdot)\right) \geq \frac{1}{n}\left(x^{*}, m\left(B_{1}\right)\right)^{+}>0
$$

which is a contradiction. Therefore $m(A)=0$ for every $A \in \Sigma$.

Remark. If $f(w) \neq 0$ for all $w \in \Omega$ then the multimeasure $F$ defined above is actually single valued.

Let $\operatorname{ext} A$ denote the extreme points of the set $A \subseteq X$.

Theorem 3.6. Let $G, M$ be as above and $f(w) \neq 0$ for every $w \in \Omega$. Then if $n \in \operatorname{ext} S_{M}$ there exists unique $m \in S_{G}$ such that $n(A)=\int_{A} f d m$ for $A \in \Sigma$ and if $n \in \operatorname{ext} S_{M}$ the measure $n$ defined by $n(A)=\int_{A} f d m$ for $A \in \Sigma$ is an extreme point of $S_{M}$.

Proof. Let $n \in S_{M}$. The existence and uniqueness of an $m \in S_{G}$ s.t. $n(A)=$ $\int_{A} f d m$ for $A \in \Sigma$ is guaranteed by Theorem 3.4 and the above remark. For the second part let $M \in S_{G}$ and $n \in S_{M}$ defined by $n(A)=\int_{A} f d m$ for $A \in \Sigma$. Suppose that $n \in \operatorname{ext} S_{M}$. Then there exist $n_{1}, n_{2} \in S_{M}, m_{1}, m_{2} \in S_{G}$, and $a \in \mathrm{IR}$ such that $n_{1}(A)=\int_{A} f d m_{1}, \quad n_{2}(A)=\int_{A} f d m_{2}$, and $n(A)=$ $a n_{1}(A)+(1-a) n_{2}(A)$ for $A \in \Sigma$. Therefore

$$
\int_{A} f d m=a \int_{A} f d m_{1}+(1-a) \int_{A} d f m_{2} \Rightarrow \int_{A} f d\left(m-a m_{1}-(1-a) m_{2}\right)=0
$$

for every $A \in \Sigma$.

By Lemma 3.5 above we get $m=a m_{1}+(1-a) m_{2}$, a contradiction which implies that $n \in \operatorname{ext} S_{M}$.

\section{REFERENCES}

1. I. Deistel and J. J. Uhl, Vector measures, Math. Surveys, vol. 15, Amer. Math. Soc., Providence, RI, 1977.

2. N. Dunford and J. Schwartz, Linear operators, Part I, Wiley Interscience, New York, 1988. 
3. C. Godet-Thobie, Some results about multimeasures and their selectors, Measure Theory at Oberwohlfach, 1979 (Kölzow, ed.), Lecture Notes in Math., vol. 794, Springer, Berlin and New York, 1979, pp. 112-116.

4. I. Kluvanek and G. Knowles, Vector measures and control systems, North-Holland, Amsterdam, 1975.

5. R. Pallu de Barriere, Multimeasures a valeurs convexes fermees, Colloque sur l'Intergration Vectoriel et Multivoque (A. Coste, ed.) expose no. 4, Caen, 1975.

6. N. S. Papagergiou, On the properties of multimeasures with values in a Banach space, Comment. Math. Univ. Carolin. (to appear).

Department of Mathematics, University of the Aegean, 83200 Karlovassi, Samos, GREECE 